\title{
Rüstzeug für die ersten Jahre
}

\author{
Programm am 8. April ab $14 \mathrm{Uhr}$
}

\section{Start-up Zahnarztpraxis - Der Weg in die Selbstständigkeit}

Referentin: Diana Brendel, M.Sc.

Der junge Zahnarzt hat viele Möglichkeiten, und genau das macht die Entscheidung so schwer. Ist die Grundsatzentscheidung zur Niederlassung getroffen, muss sich der Zahnarzt zwischen einer Neugründung und der Übernahme einer bestehenden Praxis entscheiden. Abzuwägen ist auch, ob man als „Einzelkämpfer" oder in einer Gemeinschaft arbeiten möchte.

Bereits bei dieser Entscheidungsfindung spielen wirtschaftliche Aspekte eine wesentliche Rolle. Und ist die Praxis dann eröffnet, so ist man plötzlich nicht mehr nur Zahnarzt, sondern auch Unternehmer. Ab diesem Zeitpunkt ist es unerlässlich, sich mit Managementthemen und insbesondere mit den Finanzen zu beschäftigen, denn ein betriebswirtschaftliches Grundverständnis ist die Voraussetzung für den Erfolg der eigenen Praxis.

In diesem Seminar erhalten Sie wertvolle Tipps zur Praxisgründung, erlernen das Basiswissen der Betriebswirtschaft in der Zahnarztpraxis und bekommen einen Einblick in die Unternehmensführung.

\section{Folgende Themen erwarten Sie:}

- Entscheidungshilfen zur Selbstständigkeit

- Einzelpraxis vs. Gemeinschaftspraxis

- Praxisgründung vs. Praxisübernahme

- Fakten vs. Emotionen

- Betriebswirtschaftliche Grundlagen (Gewinn/Rentabilität/ Liquidität)

- Erfolgreiche Praxisführung

- Praxiscontrolling

\subsection{Uhr: Fitnesspause auf Einladung der Deutschen Apotheker- und Ärztebank}

\subsection{Uhr: Gemeinsames Abendessen mit den Referenten}

Programm am 9. April ab $9 \mathrm{Uhr}$

Besser mit: Medizinisches und chirurgisches Basiswissen im Praxisalltag - mit praktischen Übungen

\section{Referent: Dr. Markus Tröltzsch}

\section{Programm:}

A) Medizinische Grundlagen für die orale Chirurgie - Einführung

- Die häufigsten medizinischen Herausforderungen
- Pharmakologie

- Evaluation von Patienten

B) Chirurgie Basistechniken

- Anatomie Grundlagen der Mundhöhle

- Material für verschiedene Indikationen

- Chirurgische Basistechniken

\section{Abstrakt:}

Medizinisches Wissen wird im Rahmen des zahnmedizinischen Studiums nur am Rand vermittelt. Allerdings wird dieses für den Zahnarzt immer wichtiger, da die gesundheitliche Situation der Patienten durch multiple Medikamentengaben und zahnärztlich relevante Erkrankungen immer komplexer wird. Insbesondere für Patienten bei denen parodontologische oder chirurgische Maßnahmen nötig sind, ist dieses Wissen von Nöten. Zudem werden auch chirurgische Fähigkeiten meist nur minimal vermittelt. Praxisrelevante Kenntnis von Anatomie, Sites, Schnittführung und Material muss somit häufig selbst erworben werden und steht am Anfang oft als grosses Hindernis der chirurgischen Tätigkeit im Weg.

In diesem Kurs soll medizinisches und chirurgisches Basiswissen für den Alltag vermittelt werden und sowohl auf für den Zahnarzt wichtige Pharmakologie als auch auf häufige Erkrankungen eingegangen werden. In praktischen Übungen werden zudem grundlegende chirurgische Techniken trainiert.

Ende: ca. 16:30 Uhr 